\title{
Clues to the Fundamental Nature of Gravity, Dark Energy and Dark Matter
}

\author{
Eugene Terry Tatum ${ }^{1}$, U. V. S. Seshavatharam ${ }^{2}$ \\ ${ }^{1} 760$ Campbell Ln. Ste. 106 \#161, Bowling Green, KY 42104, USA \\ ${ }^{2}$ Honorary Faculty, I-SERVE, Hyderabad, India \\ Email: ett@twc.com, seshavatharam.uvs@gmail.com
}

How to cite this paper: Tatum, E.T. and Seshavatharam, U.V.S. (2018) Clues to the Fundamental Nature of Gravity, Dark Energy and Dark Matter. Journal of Modern Physics, 9, 1469-1483.

https://doi.org/10.4236/jmp.2018.98091

Received: May 29, 2018

Accepted: July 9, 2018

Published: July 12, 2018

Copyright $(9) 2018$ by authors and Scientific Research Publishing Inc. This work is licensed under the Creative Commons Attribution International License (CC BY 4.0).

http://creativecommons.org/licenses/by/4.0/

\begin{abstract}
This paper integrates the Flat Space Cosmology (FSC) model into the Friedmann equations containing a cosmological term. The Lambda $\Lambda$ term within this model scales according to $3 H_{t}^{2} / c^{2}$ and $3 / R_{t}^{2}$. Use of the Bekenstein-Hawking definition of closed gravitational system total entropy provides for FSC cosmic parameter definitions in terms of $\sqrt{S}$. Cosmic time, radius, total matter mass-energy and vacuum energy in this model scale in exactly the same way as $\sqrt{S}$. This analysis opens the way for understanding gravity, dark energy and dark matter as being deeply connected with cosmic entropy. The recent theoretical work of Roger Penrose and Erik Verlinde is discussed in this context. The results of this FSC model analysis dovetail nicely with Verlinde's work suggesting gravity as being fundamentally an emergent property of cosmic entropy. This emergent-property-of-entropy definition of gravity, if true, would also indicate that gravitational inertia, dark matter and dark energy are simply manifestations of cosmic entropy. Thus, they would likely have no identifiable connection to quantum physics, including the standard particle model.
\end{abstract}

\section{Keywords}

Cosmology Theory, Dark Energy, Dark Matter, Cosmic Entropy, Entropic Arrow of Time, Cosmic Inflation, Black Holes, Cosmological Constant Problem, Emergent Gravity

\section{Introduction and Background}

Flat Space Cosmology (FSC) is a mathematical model of universal expansion which has proven to be remarkably accurate in comparison to observations [1] [2] [3] [4] [5]. FSC was initially developed as a heuristic mathematical model of 
the Hawking-Penrose idea that an expanding universe arising from a singularity state can be modeled as a time-reversed giant black hole. This idea was an extension of Penrose's paper [6] on the singularities of black holes and cosmology. Hawking's doctoral thesis took the idea further by proving the validity of time-reversal in the treatment of general relativity as it concerns cosmology [7]. Finally, the FSC model completes this idea by incorporating scaling black hole equations suitable for cosmology. Thus, the proven accuracy of FSC with respect to current astronomical observations does not appear to be an accident.

FSC has recently been proven to be a general relativity model by successfully integrating the FSC assumptions into the Friedmann equations which include a cosmological term and a global curvature term $k$ set to zero. The relevant equations are repeated in this paper for clarity. One of the results of integrating FSC into the Friedmann equations is that the following relation holds true in FSC:

$$
\frac{3 H^{2} c^{2}}{8 \pi G} \cong \frac{\Lambda c^{4}}{8 \pi G}
$$

This is merely a reflection that global space-time in FSC is flat during the cosmic expansion. As stipulated by the space-time curvature rules of general relativity, a globally flat universe must have a net energy density of zero. Otherwise, if the positive energy density and negative energy density terms were not equal in magnitude, there would be an observable global space-time curvature representative of the greater energy density term.

The purpose of this paper is to show how the FSC Friedmann equations evolve further from Equation (1) and what they might imply with respect to the fundamental nature of gravity, dark energy and dark matter. Before doing so, however, it is useful to review the five current assumptions of FSC and its observational correlations.

\subsection{The Five Assumptions of Flat Space Cosmology}

1) The cosmic model is an ever-expanding sphere such that the cosmic horizon always translates at speed of light $c$ with respect to its geometric center at all times $t$. The observer is defined to be at this geometric center at all times $t$.

2) The cosmic radius $R_{t}$ and total matter mass $M_{t}$ follow the Schwarzschild formula $R_{t} \cong 2 G M_{t} / c^{2}$ at all times $t$.

3) The cosmic Hubble parameter is defined to be $H_{t} \cong c / R_{t}$ at all times $t$.

4) Incorporating our cosmological scaling adaptation of Hawking's black hole temperature formula, at any radius $R_{t}$, cosmic temperature $T_{t}$ is inversely proportional to the geometric mean of cosmic total matter mass $M_{t}$ and the Planck mass $M_{p l} . R_{p l}$ is defined as twice the Planck length (i.e., as the Schwarzschild radius of the Planck mass black hole). With subscript $t$ for any time stage of cosmic evolution and subscript $p l$ for the Planck scale epoch, and incorporating the Schwarzschild relationship between $M_{t}$ and $R_{t}$, 


$$
\begin{aligned}
& k_{B} T_{t} \cong \frac{\hbar c^{3}}{8 \pi G \sqrt{M_{t} M_{p l}}} \cong \frac{\hbar c}{4 \pi \sqrt{R_{t} R_{p l}}} \\
& M_{t} \cong\left(\frac{\hbar c^{3}}{8 \pi G k_{B} T_{t}}\right)^{2} \frac{1}{M_{p l}} \\
& R_{t} \cong \frac{1}{R_{p l}}\left(\frac{\hbar c}{4 \pi k_{B}}\right)^{2}\left(\frac{1}{T_{t}}\right)^{2} \\
& R_{t} T_{t}^{2} \cong \frac{1}{R_{p l}}\left(\frac{\hbar c}{4 \pi k_{B}}\right)^{2} \\
& H_{t} \cong \frac{c}{R_{t}}
\end{aligned}
$$

5) Total entropy of the cosmic model follows the Bekenstein-Hawking black hole formula [8] [9]

$$
S_{t} \cong \frac{\pi R_{t}^{2}}{L_{p}^{2}}
$$

As previously reported [Tatum, et al (2015)], a number of past and current cosmological parameters can be calculated using the FSC model and are found to be in tight correlation with observations and the Hawking-Penrose theory. The accuracy of these correlations with observations is largely accomplished by incorporating the appropriate cosmological scaling formula for cosmic temperature [see the top equation in relation (2)]. This equation, by incorporating elementary and fundamental constants of nature, allows for FSC scaling from the Planck scale to the current scale. Thus, FSC can be considered a quantum cosmology model.

\subsection{Cosmological Parameter Derivations of FSC}

Incorporation of the FSC assumptions into the cosmological scaling temperature formula allows for the following cosmological parameter definitions. Current observational parameters are calculated in the right-hand column. The only free parameter in any of these equations is the cosmic temperature. The currently observed cosmic temperature value: $T_{0}=2.72548 \mathrm{~K}$.

$$
\begin{gathered}
R \cong \frac{\hbar^{3 / 2} c^{7 / 2}}{32 \pi^{2} k_{B}^{2} T^{2} G^{1 / 2}}, \quad R_{0} \cong \frac{\hbar^{3 / 2} c^{7 / 2}}{32 \pi^{2} k_{B}^{2} T_{0}^{2} G^{1 / 2}} \\
H \cong \frac{32 \pi^{2} k_{B}^{2} T^{2} G^{1 / 2}}{\hbar^{3 / 2} c^{5 / 2}}, \quad H_{0} \cong \frac{32 \pi^{2} k_{B}^{2} T_{0}^{2} G^{1 / 2}}{\hbar^{3 / 2} c^{5 / 2}} \\
t \cong \frac{\hbar^{3 / 2} c^{5 / 2}}{32 \pi^{2} k_{B}^{2} T^{2} G^{1 / 2}}, \quad t_{0} \cong \frac{\hbar^{3 / 2} c^{5 / 2}}{32 \pi^{2} k_{B}^{2} T_{0}^{2} G^{1 / 2}} \\
M \cong \frac{\hbar^{3 / 2} c^{11 / 2}}{64 \pi^{2} k_{B}^{2} T^{2} G^{3 / 2}}, \quad M_{0} \cong \frac{\hbar^{3 / 2} c^{11 / 2}}{64 \pi^{2} k_{B}^{2} T_{0}^{2} G^{3 / 2}} \\
M c^{2} \cong \frac{\hbar^{3 / 2} c^{15 / 2}}{64 \pi^{2} k_{B}^{2} T^{2} G^{3 / 2}},
\end{gathered}
$$




$$
H_{0}=2.167862848658891 \times 10^{-18} \mathrm{~s}^{-1}\left(66.89325791854758 \mathrm{~km} \cdot \mathrm{s}^{-1} \cdot \mathrm{Mpc}^{-1}\right)
$$

This derived current Hubble parameter value fits very closely with the low end range of the 2015 Planck Collaboration consensus observational value of 67.8 $+/-0.9 \mathrm{~km} \cdot \mathrm{s}^{-1} \cdot \mathrm{Mpc}^{-1}$

$$
t_{0} \cong \frac{1}{H_{0}}=4.612837941379141 \times 10^{17} \mathrm{~s}\left(14.61694683819266 \times 10^{9} \text { sidereal yrs }\right)
$$

(multiplying by 1 sidereal $\mathrm{yr} / 3.155814954 \times 10^{7} \mathrm{~s}$ )

$$
R_{0} \cong \frac{c}{H_{0}}=1.382894024801713 \times 10^{26} \mathrm{~m}\left(14.61720137583068 \times 10^{9} \text { light-yrs }\right)
$$

(multiplying by 1 Julian light-yr/9.4607304725808 $\times 10^{15} \mathrm{~m}$ )

This current cosmic radius value correlates with current cosmic time by $R_{o}=$ $c t_{o}$. For reasons given in the seminal FSC papers, a perpetually flat and finite space-time cosmology model has no need to incorporate a superluminal inflationary mechanism to solve the flatness and horizon problems.

$$
\begin{gathered}
V o l_{0}=\frac{4 \pi}{3}\left(\frac{c}{H_{0}}\right)^{3}=1.107784564915062 \times 10^{79} \mathrm{~m}^{3} \\
M_{0}=\frac{c^{3}}{2 G H_{0}}=9.311265291518025 \times 10^{52} \mathrm{~kg}
\end{gathered}
$$

This total matter mass number can be compared very favorably to a rough estimate made from astronomical observations. The visible matter consists of roughly 100 billion galaxies averaging roughly 100 billion stars each, of average star mass equal to roughly $1.4 \times 10^{30} \mathrm{~kg}$ (70 percent of solar mass), totaling to roughly $1.4 \times 10^{52} \mathrm{~kg}$. The 2015 Planck Collaboration report indicates a universal matter ratio of approximately 5.47 parts dark matter to 1 part visible (baryonic) matter. This brings the total estimated matter in the observable universe to approximately $9.1 \times 10^{52} \mathrm{~kg}$. A recent study [10] of average mass density of intergalactic dust gives a value of approximately $10^{-30} \mathrm{~kg} \cdot \mathrm{m}^{-3}$. Since this is approximately 1 part intergalactic dust to 1000 parts galactic and perigalactic matter, intergalactic dust does not appreciably modify the total observational estimated mass of matter given above. Accordingly, this observational estimate is remarkably close to the above FSC theoretical calculation of total cosmic matter mass. By the FSC Friedmann equations (below), the positive total matter mass-energy must always be equal in absolute magnitude to the negative dark energy. This predicts a $50 / 50$ cosmic energy density percentage ratio as opposed to the approximately $30 / 70$ ratio currently claimed by standard cosmology proponents. However, without unequivocally proving cosmic acceleration, standard cosmology cannot yet rightfully claim this 30/70 ratio. This has been discussed in numerous recent analyses of the Supernova Cosmology Project compilation data [11] [12] [13] [14] and in a recent FSC paper [15].

$$
M_{0} c^{2}=\frac{c^{5}}{2 G H_{0}}=8.368547901344209 \times 10^{69} \mathrm{~J}
$$




$$
\left.\rho_{0}=\frac{3 H_{0}^{2}}{8 \pi G}=8.405303329200976 \times 10^{-27} \mathrm{~kg} \cdot \mathrm{m}^{-3} \text { (critical mass density }\right)
$$

This closely approximates the observational critical density.

$$
\rho_{0} c^{2}=\frac{3 H_{0}^{2} c^{2}}{8 \pi G}=7.554309895973191 \times 10^{-10} \mathrm{~J} \cdot \mathrm{m}^{-3}
$$

This closely approximates the observational critical energy density and the observational vacuum energy density. They are equal in absolute magnitude in FSC.

\section{Flat Space Cosmology Friedmann Equations}

With respect to the Friedmann equations, those incorporating a non-zero cosmological term (i.e., a dark energy term) are now the most relevant since the 1998 Type Ia supernovae discoveries. Therefore, accepting Friedmann's starting assumptions of homogeneity, isotropism and an expanding cosmic system with a stress-energy tensor of a perfect fluid, we have his cosmological equation

$$
\frac{\dot{a}^{2}+k c^{2}}{a^{2}} \cong \frac{8 \pi G \rho+\Lambda c^{2}}{3}
$$

This equation is derived from the 00 component of the Einstein field equations. Since the global curvature term $k$ is always zero in FSC, Equation (9) reduces to

$$
\left(\frac{\dot{a}}{a}\right)^{2} \cong H^{2} \cong \frac{8 \pi G \rho}{3}+\frac{\Lambda c^{2}}{3}
$$

With rearrangement, we have

$$
\frac{3 H^{2}}{8 \pi G}-\frac{\Lambda c^{2}}{8 \pi G} \cong \rho
$$

This is the relevant Friedmann equation for cosmic mass density. Multiplying all terms by $c^{2}$ gives us the relevant Friedmann equation for cosmic energy density

$$
\frac{3 H^{2} c^{2}}{8 \pi G}-\frac{\Lambda c^{4}}{8 \pi G} \cong \rho c^{2}
$$

At this point it is crucial to remember that Friedmann's energy density derivation of Einstein's field equations for the cosmic system as a whole (i.e., globally) can be interpreted in the form of additive space-time curvatures represented by the individual terms. The first term can be read as the positive energy density (i.e., the positive space-time curvature) term; the second term can be read as the negative energy density (i.e., the negative space-time curvature) term; and the third term can be read as the summation (i.e., net) energy density term for global cosmic space-time curvature. Since global space-time is treated as constantly and perfectly flat in FSC, the third term must always have a net value of zero energy density. This is entirely in keeping with the general theory of relativity, as applied to cosmology, as well as current cosmological observations of flatness (i.e., 
critical density). Hence, in FSC

$$
\frac{3 H^{2}}{8 \pi G} \cong \frac{\Lambda c^{2}}{8 \pi G}
$$

And

$$
\frac{3 H^{2} c^{2}}{8 \pi G} \cong \frac{\Lambda c^{4}}{8 \pi G}
$$

From these respective critical mass density and energy density equations, it is obvious that the FSC model defines the Lambda term $\Lambda$ by

$$
\Lambda \cong \frac{3 H^{2}}{c^{2}}
$$

In FSC and other realistic linear Milne-type models, Hubble parameter $H$ is a quantity which scales with cosmic time and is defined as

$$
H \cong \frac{c}{R}
$$

where $c$ is the speed of light and $R$ is the cosmic radius as defined by the Schwarzschild formula

$$
R \cong \frac{2 G M}{c^{2}}
$$

where $M$ represents the total matter mass of the cosmic system and $G$ is the universal gravitational constant. Therefore, FSC Equation (15) substituted by equation (16) gives

$$
\Lambda \cong \frac{3}{R^{2}}
$$

So the Lambda term $\Lambda$ is also a scalar quantity (i.e., like the Hubble parameter, not actually a constant) over the great span of cosmic time. This indicates that FSC is a dynamic dark energy quintessence model.

Crucially, Equation (18) allows one to compare the Lambda term $\Lambda$ with total entropy for the FSC cosmic system over the span of cosmic time. Recalling the Bekenstein-Hawking derivation of black hole entropy [Bekenstein (1974); Hawking (1976)] as directly proportional to the event horizon surface area $\left(4 \pi R^{2}\right)$, we can apply their formula for cosmic entropy

$$
S_{t} \cong \frac{\pi R_{t}^{2}}{L_{p}^{2}}
$$

Then substituting Equation (18) into Equation (19) and rearranging terms

$$
\Lambda \cong \frac{3 \pi}{S L_{p}^{2}}
$$

Thus, the Lambda term $\Lambda$ in FSC is inversely proportional to total cosmic entropy $S$ at all times. Substituting Equation (20) into Equation (15) gives

$$
S \cong \frac{\pi c^{2}}{H^{2} L_{p}^{2}}
$$


and

$$
H \cong \frac{c}{L_{p}} \sqrt{\frac{\pi}{S}}
$$

And, since the reciprocal of the Hubble parameter is the measure of cosmic time $t$ in FSC

$$
t \cong \frac{L_{p}}{c} \sqrt{\frac{S}{\pi}}
$$

So cosmic time is always directly proportional to $\sqrt{S}$, with entropy $S$ as defined by Bekenstein and Hawking. Thus, the "entropic arrow of time" is clearly defined in the FSC model.

The dark energy density cosmological term is not only expressed as $\left(\Lambda c^{4} / 8 \pi G\right)$ in FSC Friedmann Equation (14) but, by incorporating equation (20) into this term, we now have a dark energy density equation

$$
\frac{\Lambda c^{4}}{8 \pi G} \cong \frac{3 c^{4}}{8 G S L_{p}^{2}} \cong \frac{3 H^{2} c^{2}}{8 \pi G}
$$

where in any of these terms can be used interchangeably to quantify the absolute magnitude of the cosmic dark energy density at all times.

Given the above relations, simple algebraic rearrangements allow for expressions of the following FSC parameters in terms of $\sqrt{S}$

$$
\sqrt{S}=\frac{c \sqrt{\pi}}{L_{p}} t
$$

Showing direct proportionality between cosmic entropy and cosmic time $t$.

$$
\sqrt{S}=\frac{\sqrt{\pi}}{L_{p}} R
$$

Showing direct proportionality between cosmic entropy and cosmic radius $R$.

$$
\sqrt{S}=\frac{2 G \sqrt{\pi}}{c^{2} L_{p}} M
$$

Showing direct proportionality between cosmic entropy and total cosmic matter mass $M$.

$$
\sqrt{S}=\frac{\hbar c^{5}}{32 \pi^{\frac{3}{2}} k_{B}^{2} G} T_{U}^{-1}=\frac{\hbar c^{5}}{32 \pi^{\frac{3}{2}} k_{B}^{2} G} T^{-2}
$$

Showing indirect proportionality between cosmic entropy and cosmic temperatures $T_{U}$ and $T$.

$$
\sqrt{S}=\frac{c \sqrt{\pi}}{L_{p}} H^{-1}
$$

Showing indirect proportionality between cosmic entropy and Hubble parameter $H$. 


$$
\sqrt{S}=\frac{\sqrt{3 \pi}}{L_{p}} \Lambda^{-\frac{1}{2}}
$$

Showing indirect proportionality between cosmic entropy and cosmic Lambda.

$$
\begin{gathered}
\sqrt{S}=\frac{2 G \sqrt{\pi}}{c^{4} L_{p}} M c^{2} \\
\sqrt{S}=\frac{2 G \sqrt{\pi}}{c^{4} L_{p}}(V \cdot E)
\end{gathered}
$$

Showing direct proportionality between cosmic entropy and total cosmic matter mass-energy and negative vacuum energy.

$$
\frac{M}{R}=\frac{c^{2}}{2 G}
$$

Showing the Schwarzschild relation between total cosmic matter mass $M$ and radius $R$.

$$
\frac{G M^{2}}{R^{2}}=\frac{c^{4}}{4 G}
$$

Showing an FSC Newtonian gravitational force relation based upon the Schwarzschild relation.

$$
\begin{aligned}
& M c^{2}=\frac{c^{4}}{2 G} R \\
& |V \cdot E|=\frac{c^{4}}{2 G} R
\end{aligned}
$$

Showing FSC energy definitions of total cosmic matter mass-energy and vacuum energy.

$$
\begin{gathered}
\frac{M c^{2}}{2}=\frac{G M^{2}}{R^{2}} R \\
\frac{|V \cdot E|}{2}=\frac{G M^{2}}{R^{2}} R
\end{gathered}
$$

Showing FSC matter mass-energy and vacuum energy relations with FSC Newtonian gravitational work (incorporating $E=M c^{2}$, of course).

$$
\frac{M c^{2}}{2}+\frac{V \cdot E}{2}=\left(\frac{G M^{2}}{R^{2}}\right) R-\left(\frac{G M^{2}}{R^{2}}\right) R=0
$$

Showing how conservation of energy works in the expanding FSC closed energy system. Such a spatially flat cosmic system, if it begins with net zero energy, must always be at net zero energy.

\section{Discussion}

Incorporation of the FSC assumptions into the Friedmann equations containing a cosmological term provides unique insights into the possible nature of gravity, 
dark energy and dark matter. The cosmological term is usually expressed in the form of a negative energy density in counterbalance to the positive energy density of total matter (baryonic plus dark matter). Given the recent discovery of dark energy [16] [17] [18], and in the context of general relativity, dark energy is believed to represent a systemic negative gravitational energy within the cosmological vacuum. It seems reasonable to assume that dark energy and the negative vacuum energy represented by Friedmann's cosmological term are one and the same. The important question concerns whether dark energy is a completely new physical entity or one which we already know by another name.

Gravitational energy within the vacuum of a closed gravitating system has long been known to be a negative energy. For an excellent discusson as to why gravitational energy, in the form of potential energy, must be a negative energy in comparison to matter energy, the interested reader is referred to pages $11-14$ and 289 - 293 in Alan Guth's excellent book entitled, "The Inflationary Universe" [19]. Gravitational systems perform work on mass bodies when aggregating them. Thus, by $E=m c^{2}$, all aggregating bodies acquire additional increments of mass corresponding to their newly-acquired energy. By convention, this is regarded as a gain in the positive energy of matter. However, the generalized vacuum part of any such closed system must gain an equal amount of negative energy during all such gravitational interactions, in order to obey the Law of Conservation of Energy. No net energy can be gained or lost by a gravitating closed system, whether it is expanding, contracting or fixed in radius. Thus, increasingly negative gravitational energy of the vacuum becomes a strong candidate for dark energy.

In this context, it is easy to understand the meaning of FSC mass density and energy density Equations (13) and (14), respectively. Equality between these total matter and vacuum energy terms is mandatory in a closed system such as FSC. And, because these terms are of opposite signs with respect to their energy densities, the net global energy density of a spatially flat closed gravitating system must be perpetually zero from inception. The FSC assumptions, by virtue of the Schwarzschild formula relationship between total matter mass $M_{t}$ and radius $R_{t}$, and by virtue of the Hubble parameter definition as $c / R_{t}$ create a flat universe perpetually at the Friedmann critical energy density of $\left(3 H^{2} c^{2} / 8 \pi G\right)$. By incorporating the Schwarzschild relation [Equation (32)] into total matter and vacuum energy Equations (34A) and (34B), one can readily see how Newtonian gravitational work (now slightly modified by incorporating $E=m c^{2}$, of course) can be expressed in Equations (35A) and (35B). Incorporating the correct negative energy signage of vacuum energy (i.e., dark energy) into Equation (36) shows how a closed net zero energy (i.e., flat) gravitating universe could evolve from a net zero energy quantum fluctuation event.

In sharp contrast to FSC, standard inflationary cosmology has an entirely different explanation for cosmological flatness in universal observations going all the way back to the very early universe [the Cosmic Microwave Background $(\mathrm{CMB})$ radiation was released before 3 one-hundred-thousandths $(0.0000277)$ of 
the current age of the universe]. Standard cosmology maintains that a quantum fluctuation event within a zero energy pre-Big Bang state kicked off the universal expansion. It also maintains that gravity was the first of four fundamental forces to "freeze out" following an exceedingly brief exponential inflationary phase. Standard model cosmologists believe our current universe to contain an extremely small net negative energy. In other words, they believe in cosmic acceleration (as opposed to constant velocity light speed expansion), despite these current observations of extreme flatness. However, if our universe began from a zero energy state and now has a non-zero energy density, however small, this would appear to violate conservation of energy! Furthermore, one must ask what kind of force drove the inflationary ("inflaton") field if gravity did not already exist at the inception of the universe. Cosmic inflation energy appears to be suspiciously like early cosmic dark energy, which must be negative gravitational energy in nature. The Big Bang theory is derived from general relativity, which is entirely a gravity theory. To require that a gravity theory incorporate a pre-gravity phase within its cosmology, however brief in duration, sounds very much like nonsense. Moreover, cosmic inflation is an ad hoc theory "...contrived with the goal of arranging for the density perturbations to come out right" [Guth (1997), page 238]. Cosmic inflation, in its many different ad hoc forms, appears to be a deeply flawed theory, as nicely elaborated by one of its founders [20].

The purpose of this paper, however, is not to explain why the FSC model, now integrated into the flat universe Friedmann equations with a cosmological term, rigorously follows observations of cosmic flatness within the $\mathrm{CMB}$. This point has been made in previous FSC publications [Tatum (2015)]. Rather, it is the purpose of this paper to further explore the possible nature of gravity, dark energy and dark matter. While the FSC model clearly indicates that dark energy is systemic negative gravitational energy, the key question becomes "How does this finite constant velocity expanding cosmic system work at its most fundamental level? Specifically, what is the fundamental nature of its gravity, especially in relation to dark energy and dark matter?"

Possible clues to the fundamental nature of gravity and dark energy are provided in the new FSC Friedmann equations incorporating a cosmic entropy term. In Equation (18) Lambda term $\Lambda$ is always inversely proportional to the square of the cosmic radius. Thus, Lambda scales approximately 121.26 base 10 orders of magnitude from the Planck scale. Interestingly, $10^{121}$ is the magnitude of the "cosmological constant problem" [21] [22]. Furthermore, Equation (18) is seen (in rearranged form) on page 277 of Roger Penrose's latest book [23], if one assumes the standard $4 \pi R^{2}$ formula for the cosmic horizon surface area $A_{\text {cosm }}$. Notably, this equation occurs in Penrose's discussion of cosmic entropy, which assumes the Bekenstein-Hawking definition of cosmic entropy [see FSC Equation (19)]. So, while Lambda in general relativity is assumed to be a constant by proponents of standard cosmology, the FSC model and Penrose clearly indicate Lambda to be a declining scalar of negative energy density in an expanding 
closed general relativity model. Lambda is clearly an inverse scalar of cosmic entropy, as best seen in FSC Equation (20). However, most importantly, as seen by integrating Equation (26) into (34B), total vacuum energy scales in direct proportion to total cosmic entropy, specifically in the form of $\sqrt{S}$. One must keep in mind that the Bekenstein-Hawking definition of cosmic entropy is a unit-less ratio, so $\sqrt{S}$ is also a measure of cosmic entropy, but on a scale identical to that of the other scaling FSC parameters. Figure 1 and Figure 2 below graphically show the intimate relationship between scaling FSC parameters and total cosmic entropy term $\sqrt{S}$. It is entirely appropriate to use $\sqrt{S}$ as a cosmological clock because Equation (25) clearly demonstrates that FSC models the "entropic arrow of time." Notice also that the recently-introduced FSC "Universal Temperature" $T_{u}$ [24] inversely scales to the same degree as $\sqrt{S} \quad$ (60.63 logs of 10 from the Planck scale). $T_{u}$ has a direct one-to-one correspondence to the $\mathrm{T}$ Kelvin temperature scale by the Universal Temperature definition, $T_{u}=T^{2}$.

This idea that total cosmic entropy can be regarded as a cosmological clock is not entirely new, although the FSC model clearly indicates the similarly scaling entropy clock to be in the form of $\sqrt{S}$. Furthermore, the FSC Friedmann entropy equations introduced in this paper clearly point to cosmic entropy being fundamental to the nature of gravity. Penrose introduces the concept of gravitational entropy to readers on page 256 of his book. Gravitational entropy differs significantly from the entropy of an equilibrated ideal gas, wherein maximum average particle separation at a given temperature characterizes the maximum entropy state. In contrast, in a gravitating universe, the ongoing clustering of stars and galaxies, and particularly black holes, is in the direction of greater gravitational entropy! This is made abundantly clear by comparing deep space observational astronomy with observations of (approximately) co-moving galaxies. Supermassive black holes, in particular, are now thought to be huge repositories of total cosmic entropy.

A review of the possible fundamental nature of gravity with respect to cosmic entropy should begin with a landmark paper by Erik Verlinde [25]. In this paper, Verlinde makes a very persuasive argument that cosmic entropy manifests itself as gravity! He shows in great detail, by a heuristic approach, how gravity could well be an emergent property of cosmic entropy. In other words, at the quantum level, our conventional conception of gravity as a fundamental force might be just as meaningless as a conception of consciousness within two connecting neurons. Emergent properties are most evident in complex systems with high degrees of freedom. They are difficult, if not impossible, to observe at the smallest scales. This could very well nullify the assumption of string theorists that gravity should ultimately be definable fundamentally at the quantum scale. This does not bode well for a "quantum gravity" theory to be any different from FSC "quantum cosmology" as first presented in 2015 and now presented in its final form in the present paper.

If Verlinde's compelling emergent property argument ultimately prevails, gravitational inertia (including that of dark matter!) and dark energy would also be 


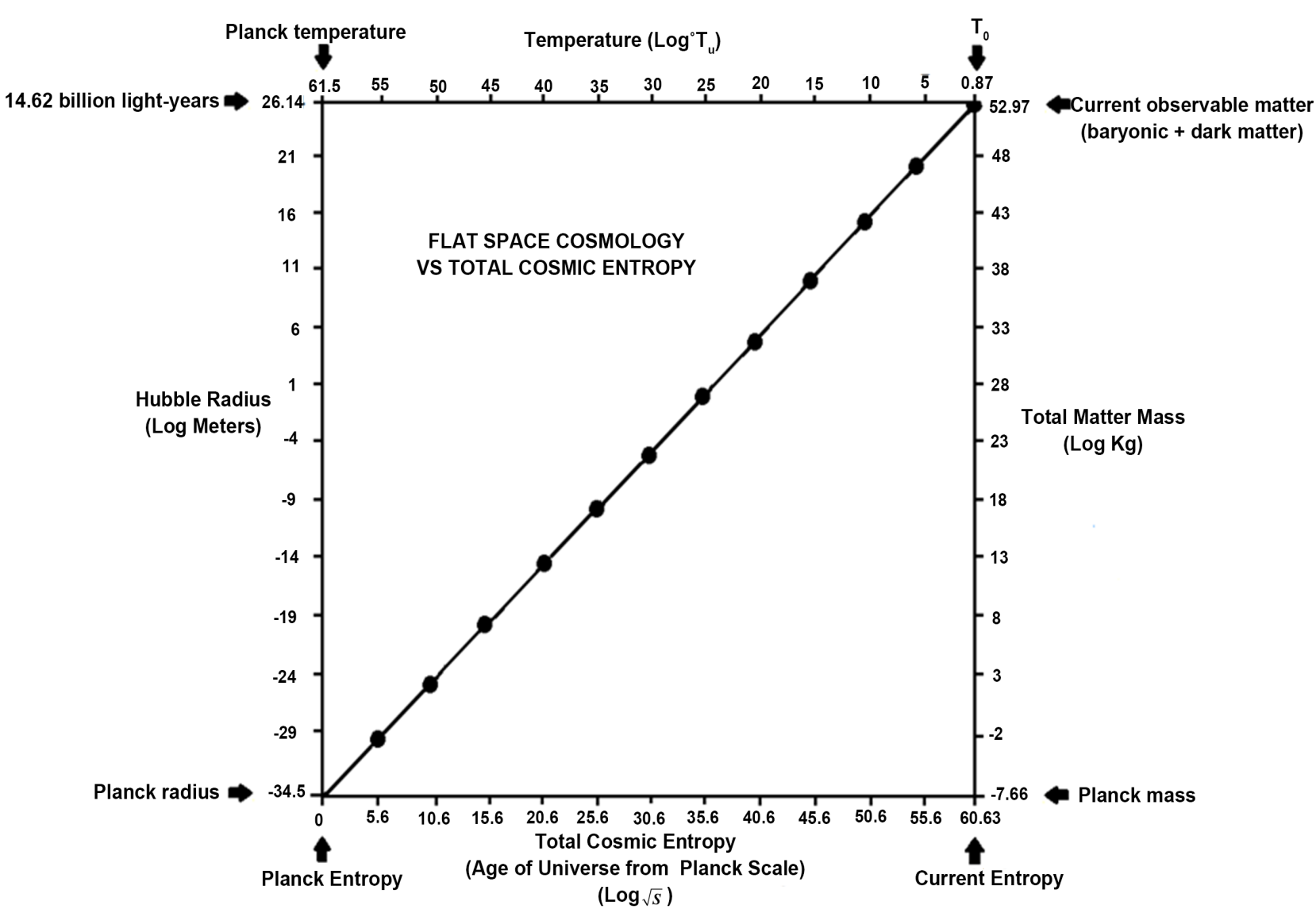

Figure 1. Universal Temperature $T_{u}$, Radius and Total Matter Mass vs. Entropy.

emergent properties of cosmic entropy. The total matter mass attributable to gravitational entropy, by equations (27) and (31A), must include dark matter. This may well explain why dark matter does not integrate into the standard particle model. The majority of the dark matter yet to be discovered may not, in fact, be particulate, but rather the previously unaccounted for entropic gravitational inertia of visible matter.

Verlinde's heuristic approach to a fundamental understanding of gravity as an emergent property of cosmic entropy dovetails nicely with this updated FSC cosmology model. FSC also began as a heuristic model, as did Einstein's (and Planck's) photon. Importantly, neither approach relies in any way on a curved (i.e., non-flat) geometrical description of gravity. Although general relativity's curved space-time is a supremely accurate and beautiful geometrical description of gravity, it is not fundamental to the nature of gravity. W.S. Krogdahl, for instance, achieved a similarly accurate mathematical model of gravity and cosmology in flat space-time by starting his development with the integration of $E=$ $m c^{2}$ into Newtonian gravity [26] [27] [28]. Krogdahl's approach appears to be vindicated by Equations (33) thru (36) in the present paper. These equations also give meaning to Newton's discovery that the force of gravity is inversely proportional to $R^{2}$ ! 


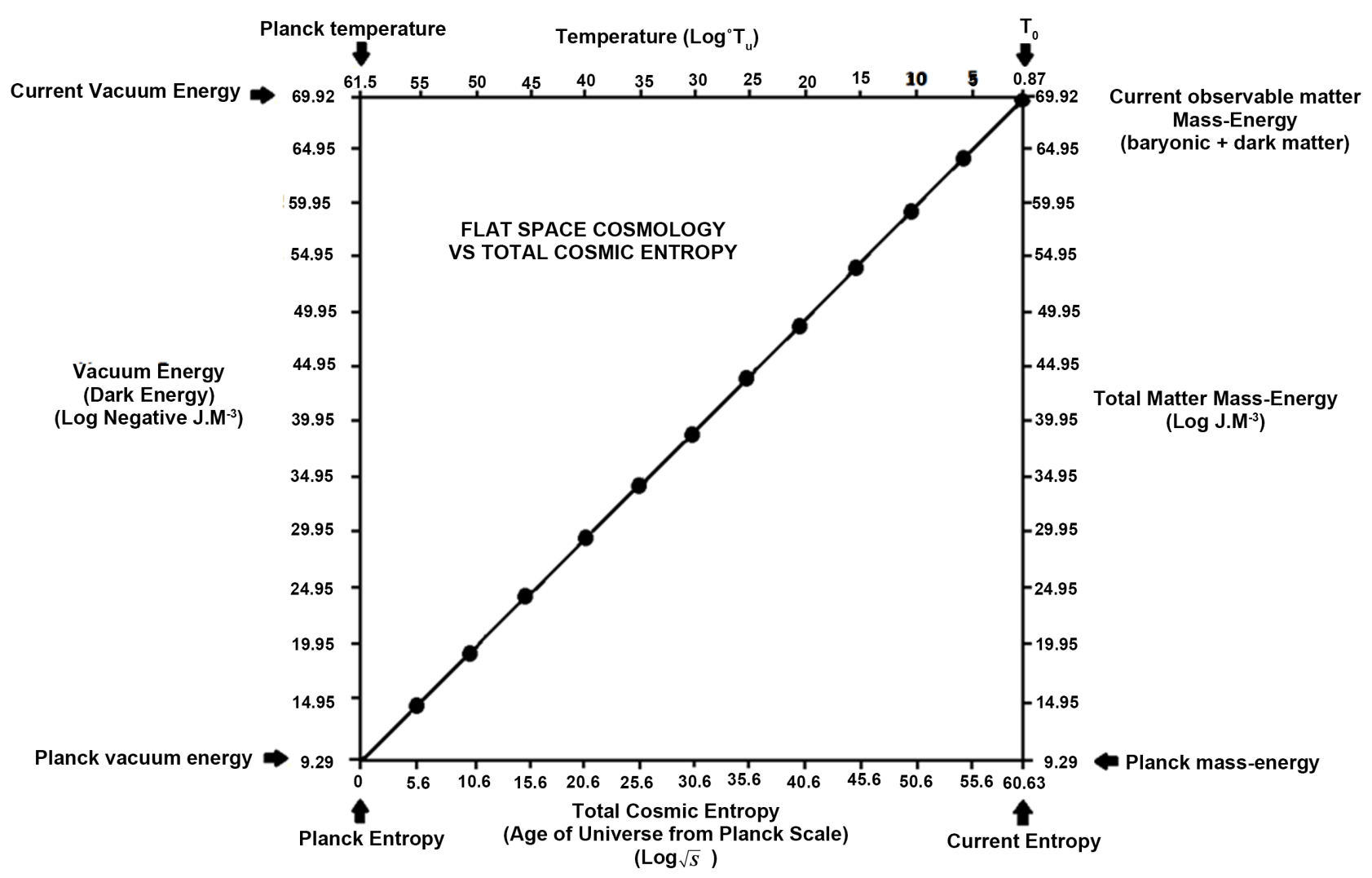

Figure 2. Universal temperature $T_{u}$, vacuum energy and total mass-energy vs. entropy.

\section{Summary and Conclusions}

The purpose of this paper has been to integrate the highly successful FSC model assumptions into the Friedmann equations in an effort to explore the fundamental nature of gravity, dark matter and dark energy. In doing so, there should be no doubt that the results are within the rules of general relativity, particularly in light of the fact that black hole equations already follow the rules of general relativity.

The results of this exercise are quite intriguing. The Lambda term $\Lambda$ in FSC must follow Equations (15) and (18), indicating that FSC is a dynamic scalar dark energy (quintessence) model of the $w C D M$ type (the FSC equation of state term $w$ is perpetually -1.0). Lambda is shown to be a declining scalar of negative gravitational vacuum energy density (i.e., dark energy density). However, Lambda is an inverse scalar of total cosmic entropy $S$ by Equations (20) and (30). Total matter mass-energy, vacuum energy and cosmic time are shown to be directly proportional to total cosmic entropy in the form of $\sqrt{S}$. Thus, the "entropic arrow of time" is clearly demonstrated in FSC, and $\sqrt{S}$ can be used as the FSC time clock, due to the direct proportionality shown in Equation (25) between cosmic entropy $\sqrt{S}$ and cosmic time $t$ (see Figure 1 and Figure 2).

A search of recent literature concerning the possible relationship between total cosmic entropy and gravitational interactions identifies Roger Penrose, Stephen Hawking, and Erik Verlinde as pioneers in this field. Penrose's book shows 
how gravitational entropy clearly differs from the entropy of an ideal gas in the sense that gravitational clustering in the form of stars, galaxies and black holes, is representative of a high (gravitational) entropy state. Black holes, in particular, are huge reservoirs of entropy in its highest possible state. In fact, the FSC model, in this context of gravitational entropy, clearly indicates that black holes may be equivalently defined as localized zones of maximum possible gravitational entropy, from the Planck scale to the scale of the current universe.

Verlinde's paper on the origin of gravity shows very clearly how gravity could be an emergent property of total cosmic entropy. If so, then gravity may be no more definable at the quantum level than consciousness can be defined within two connecting neurons. Moreover, if gravity is truly an emergent property of total cosmic entropy, then it existed from the inception of universal expansion, as opposed to "freezing out" after a pre-gravity inflationary phase. This emergent property of entropy concept would also indicate that all gravitational manifestations, including gravitational inertia, dark matter and dark energy, are emergent properties, with no likely identifiable connection to quantum physics, including the standard particle model.

\section{Dedications and Acknowledgements}

Both authors dedicate this paper to Dr. Stephen Hawking and Dr. Roger Penrose for their groundbreaking work on black holes and their possible application to cosmology. Dr. Tatum thanks Dr. Rudolph Schild of the Harvard Center for Astrophysics for his past support and encouragement. Author Seshavatharam UVS is indebted to professors Brahmashri M. Nagaphani Sarma, Chairman, Shri K.V. Krishna Murthy, founding Chairman, Institute of Scientific Research in Vedas (I-SERVE), Hyderabad, India, and to Shri K.V.R.S. Murthy, former scientist IICT (CSIR), Govt. of India, Director, Research and Development, I-SERVE, for their valuable guidance and great support in developing this subject.

\section{References}

[1] Tatum, E.T., Seshavatharam, U.V.S. and Lakshminarayana, S. (2015) International Journal of Astronomy and Astrophysics, 5, 116-124. https://doi.org/10.4236/ijaa.2015.52015

[2] Tatum, E.T., Seshavatharam, U.V.S. and Lakshminarayana, S. (2015) Journal of Applied Physical Science International, 4, 18-26.

[3] Tatum, E.T., Seshavatharam, U.V.S. and Lakshminarayana, S. (2015) Frontiers of Astronomy, Astrophysics and Cosmology, 1, 98-104.

http://pubs.sciepub.com/faac/1/2/3

[4] Tatum, E.T., Seshavatharam, U.V.S. and Lakshminarayana, S. (2015) International Journal of Astronomy and Astrophysics, 5, 133-140. https://doi.org/10.4236/ijaa.2015.53017

[5] Planck Collaboration XIII (2016) Astronomy \& Astrophysics, 594, A13. http://arxiv.org/abs/1502.01589

[6] Penrose, R. (1965) Physical Review Letters, 14, 57. 
https://doi.org/10.1103/PhysRevLett.14.57

[7] Hawking, S. and Penrose, R. (1970) Proceedings of the Royal Society of London. Series $A$, 314, 529-548. https://doi.org/10.1098/rspa.1970.0021

[8] Bekenstein, J.D. (1974) Physical Review D, 9, 3292-3300. https://doi.org/10.1103/PhysRevD.9.3292

[9] Hawking, S. (1976) Physical Review D, 13, 191-197. https://doi.org/10.1103/PhysRevD.13.191

[10] Inoue, A.K. (2004) Monthly Notices of the Royal Astronomical Society, 350, 729-744. https://doi.org/10.1111/j.1365-2966.2004.07686.x

[11] Tutusaus, I., et al. (2017) Is Cosmic Acceleration Proven by Local Cosmological Probes? Astronomy \& Astrophysics, 602_A73. arXiv:1706.05036v1 [astro-ph.CO].

[12] Nielsen, J.T., et al. (2015) Marginal Evidence for Cosmic Acceleration from Type Ia Supernovae. arXiv:1506.01354v1.

[13] Wei, J.-J., et al. (2015) Astronomical Journal, 149, 102-112. https://doi.org/10.1088/0004-6256/149/3/102

[14] Melia, F. (2012) Astronomical Journal, 144. arXiv:1206.6289 [astro-ph.CO]. https://doi.org/10.1088/0004-6256/144/4/110

[15] Tatum, E.T. and Seshavatharam, U.V.S. (2018) Journal of Modern Physics, 9, 1397-1403. https://doi.org/10.4236/jmp.2018.97084

[16] Perlmutter, S., et al. (1999) Astrophysical Journal, 517, 565-586. https://doi.org/10.1086/307221

[17] Schmidt, B., et al. (1998) Astrophysical Journal, 507, 46-63. https://doi.org/10.1086/306308

[18] Riess, A.G., et al. (1998) Astronomical Journal, 116, 1009-1038. https://doi.org/10.1086/300499

[19] Guth, A.H. (1997) The Inflationary Universe. Basic Books, New York.

[20] Steinhardt, P.J. (2011) Scientific American, 304, 18-25. https://doi.org/10.1038/scientificamerican0411-36

[21] Weinberg, S. (1989) Reviews of Modern Physics, 61, 1-23. https://doi.org/10.1103/RevModPhys.61.1

[22] Carroll, S. (2001) Living Reviews in Relativity, 4, 5-56. https://doi.org/10.12942/lrr-2001-1

[23] Penrose, R. (2016) Fashion Faith and Fantasy in the New Physics of the Universe. Princeton University Press, Princeton. https://doi.org/10.1515/9781400880287

[24] Tatum, E.T. and Seshavatharam, U.V.S. (2018) Journal of Modern Physics, 9, 1404-1414. https://doi.org/10.4236/jmp.2018.97085

[25] Verlinde, E. (2010) On the Origin of Gravity and the Laws of Newton. arXiv: $1001.0785 \mathrm{v} 1$

[26] Krogdahl, W.S. (2006) Cosmology in Flat Space-Time. arXiv:gr-qc/0402016v3

[27] Tatum, E.T. (2017) Journal of Modern Physics, 8, 2087-2095. https://doi.org/10.4236/jmp.2017.813127

[28] Tatum, E.T. (2017) Journal of Modern Physics, 8, 2096-2103. https://doi.org/10.4236/jmp.2017.813128 